\title{
New results of the antiproton-carbon annihilation cross section measurement at low energies
}

\author{
H. Aghai-Khozani ${ }^{1}$, M. Corradini ${ }^{2,3}$, R. Hayano ${ }^{4}$, M. Hori ${ }^{1,4}$, M. Leali ${ }^{2,3}$, E. Lodi-Rizzini ${ }^{2,3}$, \\ V. Mascagna ${ }^{2,3, \star}$, Y. Murakami ${ }^{4}$, M. Prest ${ }^{5,6}$, L. Solazzi ${ }^{7,3}$, E. Vallazza ${ }^{8}$, L. Venturelli $i^{2,3}$, and \\ H. Yamada 4 \\ ${ }^{1}$ Max-Planck-Institut für Quantenoptik, D-85748 Garching, Germany \\ ${ }^{2}$ Dipartimento di Ingegneria dell'Informazione, Università degli Studi di Brescia, I-25123 Brescia, Italy \\ ${ }^{3}$ Istituto Nazionale di Fisica Nucleare - Pavia, I-27100 Pavia, Italy \\ ${ }^{4}$ Department of Physics, University of Tokyo, Bunkyo-ku, Tokyo 113-0033, Japan \\ ${ }^{5}$ Dipartimento di Scienza e Alta Tecnologia, Università degli Studi dell'Insubria, I-21100 Varese, Italy \\ ${ }^{6}$ Istituto Nazionale di Fisica Nucleare - Milano Bicocca, I-20126 Milano, Italy \\ ${ }^{7}$ Dipartimento di Ingegneria Meccanica e Industriale, Università degli Studi di Brescia, I-25123 Brescia, Italy \\ ${ }^{8}$ Istituto Nazionale di Fisica Nucleare - Trieste, I-34127 Trieste, Italy
}

\begin{abstract}
The antinucleon-nuclei annihilation cross section at very low energy has been measured at the LEAR facility (CERN) during the 80's and 90's and recently at the Antiproton Decelerator. The results have raised some interesting questions that still need answers. The ASACUSA collaboration performed an experiment to measure the $\sigma_{\text {ann }}$ of $100 \mathrm{MeV} / \mathrm{c}$ antiprotons on carbon target and the preliminary results are presented here.
\end{abstract}

\section{Introduction}

The interest for the very low energy $\bar{p}$ - and $\bar{n}$-nuclei interaction is due to their influence on both fundamental cosmology and nuclear physics. The study of the annihilation cross section on matter can give some contribution to the universe matter-antimatter asymmetry puzzle, as well as helping in the definition of strong interaction model parameters.

So far, the collected data for the antiproton and antineutron cross section show some discrepancies: at momenta of 300-400 MeV/c they both result [1] larger than predicted by the optical potential model which well reproduces the existing data at higher energies [2]. Moreover, the $\bar{n}$ annihilation cross section on several nuclei measured in the $70-400 \mathrm{MeV} / \mathrm{c}$ momentum range [1] seems to follow a $1 / \mathrm{p}^{2}$ trend rather than a $1 / \mathrm{p}$ that is the expected one for a neutral projectile.

A comparison between the $\bar{p}$ and $\bar{n}$ data can represent a step forward in the clarification of the unsolved issues. Antiproton annihilation cross section data on proton are available for momenta below $80 \mathrm{MeV} / \mathrm{c}$ and on $\mathrm{Sn}$ for $100 \mathrm{MeV} / \mathrm{c}$ [3]. The ASACUSA Collaboration recently performed the measurement of the antiproton annihilation cross section in the $\sim 100 \mathrm{MeV} / \mathrm{c}$ momentum region and the first preliminary results will be shown here.

^e-mail: valerio.mascagna@unibs.it 


\section{Experimental apparatus and technique}

The experimental setup is presented in Fig. 1. A vacuum chamber composed by two cylindrical sections (total length $300 \mathrm{~cm}$, diameters $120 \mathrm{~cm}$ and $60 \mathrm{~cm}$ ) is put into the existing ASACUSA beam line which is equipped with two GEM profile beam monitors for the beam alignment and focusing. A system of 4 movable holders allows the positioning of $13 \mathrm{~mm}$ diameter circular frames (with or without targets) on the beam axis in different positions [4]. The targets consist in very thin selfsupporting diamond-like carbon layers by Micromatter ${ }^{1}$ with a thickness of $700 \mathrm{~nm}$ and $1000 \mathrm{~nm}$.

Outside the chamber, in a position corresponding to the target, an arrangement of planar detectors is installed to detect the passage of the charged particles from $\bar{p}$ annihilations. They are made of plastic scintillator bars readout by multi-anode PMTs and a custom designed electronics system [6]. A Cherenkov counter is placed at the end of the beam line where all the $\bar{p}$ s annihilate.

Bunches of $\sim 5 \times 10^{6}$ antiprotons, $50 \mathrm{~ns}$ long (rise time $\sim 15 \mathrm{~ns}$ ) are provided by the AD at a kinetic energy of $5.3 \mathrm{MeV}$. The $\bar{p}$ s travel in the vacuum chamber at a speed of $3 \mathrm{~cm} / \mathrm{ns}$ and almost all of them arrive at the end of the beam line after $\sim 120 \mathrm{~ns}$, while a small fraction, of the order of 1-10 every $10^{6}$ incoming, can undergo in-flight annihilation in the target (the physics process under study). The $\bar{p}$ s scattered on the lateral walls can represent a background for the measurement. Actually their annihilation is delayed of more than $20 \mathrm{~ns}$ with respect to the early annihilations in the target since the chamber radius is $60 \mathrm{~cm}$. Therefore, a fiducial time interval to count the annihilation events is selected: it corresponds to the first 20 ns region of the annihilations.

The data acquisition system performs a sampling at $300 \mathrm{MHz}$ of the scintillator channel signal providing a hit time profile for every crossing bunch (see Fig. 2a). The time resolution at the ns level of the acquisition chain is enough to distinguish the hits coming from the target region and the ones from the chamber walls (time separation of $\geq 20 \mathrm{~ns}$ ).

The calibration of the Cherenkov detector which monitors the beam intensity is performed with dedicated runs: a second circular empty frame is put $15 \mathrm{~cm}$ downstream of the target in order to intercept a known angular fraction of the scattered $\bar{p}$ s that can be calculated using the Rutherford cross section and compared to the acquired data in this configuration.

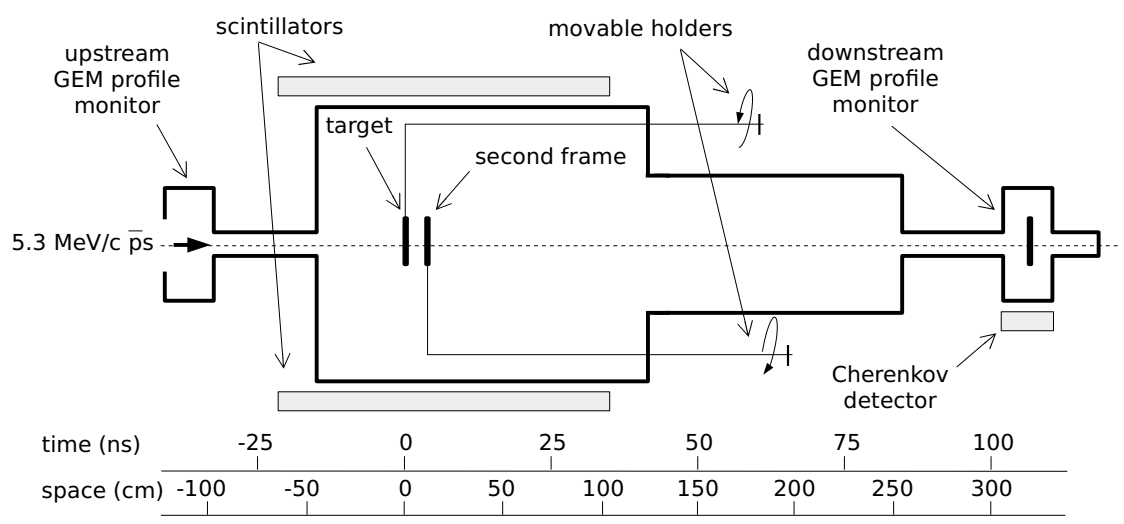

Figure 1: Scheme of the experimental setup. The time scale is based on $\bar{p}$ speed of $3 \mathrm{~cm} / \mathrm{ns}$.

\footnotetext{
${ }^{1}$ http://www.micromatter.com/dlc.php
} 


\section{Results}

The plots in Fig. 2a show the time profiles, for different types of runs, detected while the $\bar{p}$ bunch crosses the target. The time separation between the annihilation in the target region (defined as the $520-540 \mathrm{~ns}$ window), from the lateral walls (after $20 \mathrm{~ns}$ or more) and from the end wall (after $100 \mathrm{~ns}$ ) is possible. Our measurements lead to a preliminary evaluation of: $\sigma_{a n n}=2.32 \pm 0.35$ barn (statistical contribution to the error is $6 \%$ ). Fig. $2 \mathrm{~b}$ presents the obtained result superimposed to the existing $\bar{p}$ and $\bar{n}$ annihilation data on carbon [1,7] and calculation from [2]. The total error can be reduced from $\sim 15 \%$ to $\sim 10 \%$ by implementing a better synchronization in the detector data.

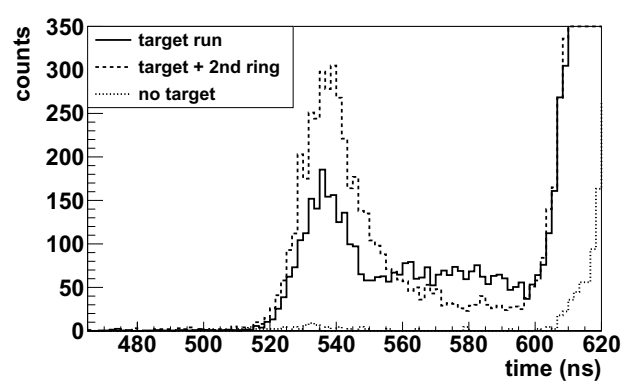

(a)

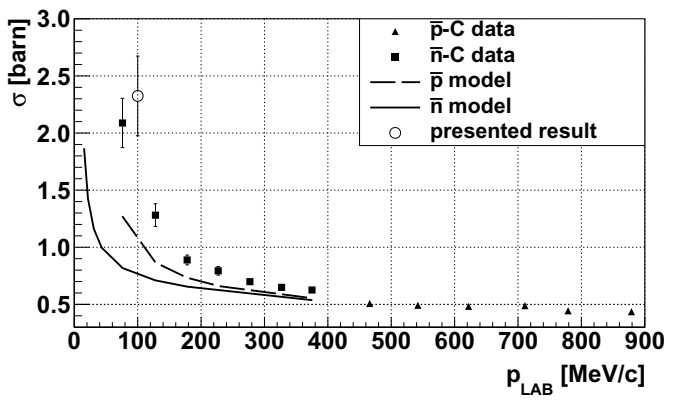

(b)

Figure 2: (a) Normalized hit profiles of one of the scintillator plane for different runs. (b) Result of the $\bar{p}$ annihilation cross section on carbon measurement superimposed to the existing data for $\overline{\mathrm{p}}, \overline{\mathrm{n}}$ and the corresponding theoretical predictions [2].

\section{Conclusions}

We reported the preliminary result for the $\bar{p}$-C annihilation cross section measurements at $5.3 \mathrm{MeV}$. The number is larger (1.5 times) than the $\bar{n}-\mathrm{C}$ one as expected by a Coulomb focusing mechanism; nevertheless it is twice the optical model prediction [2], a still unexplained issue (the same occurs in the $\bar{n}$ case). In the near future, when the ELENA facility will be operative at the AD, the same measurements will be possible at $100 \mathrm{keV}$ as already demonstrated in [8].

\section{References}

[1] M. Astrua et al., Nucl. Phys. A 697, 209 (2002)

[2] E. Friedman et al., Nucl. Phys. A 925, 141 (2014)

[3] A. Bianconi et al., Phys. Lett. B 704, 461 (2011)

[4] M. Corradini et al., Nucl. Instr. Meth. A 711, 12 (2013)

[5] H. Aghai-Kohzani et al., submitted to J. Phys. Soc. Jpn. Conf. Proceedings of LEAP 2016

[6] M. Corradini et al., Hyp. Int. 233, 53 (2015)

[7] K. Nakamura et al., Phys. Rev. Lett. 52, 731 (1984)

[8] H. Aghai-Kohzani et al., Eur. Phys. J. Plus 127, 55 (2012) 